\title{
Smoking habits and the risk of type 2 diabetes mellitus in women
}

\author{
Lina Radzeviciene', Rytas Ostrauskas' ${ }^{1}$, Evelina Praneviciene ${ }^{2}$
}

\begin{abstract}
${ }^{1}$ Institute of Endocrinology, Medical Academy, Lithuanian University of Health Sciences;
${ }^{2}$ Faculty of Medicine, Medical Academy, Lithuanian University of Health Sciences.
\end{abstract}

\section{Objectives}

Diabetes mellitus is one of the biggest public health concerns. The emerging pandemic number of patients with type 2 diabetes is driven by the combined effects of population ageing, rising levels of obesity and inactivity [1]. Type 2 diabetes appears to involve interaction between susceptible genetic backgrounds and environmental factors [2]. Smoking exacts a devastating damage on public health. Tobacco use is the most important cause of preventable morbidity and mortality around the world [3]. It's important to identify modifiable risk factors for type 2 diabetes, which may help reduce the risk of the disease. The aim of the study was to assess the relationship between smoking and the risk of type 2 diabetes in women.

\section{Methods}

In case-control study were included 168 cases aged 35-85 years with a newly confirmed diagnosis of type 2 diabetes mellitus during the one whole year and 336 controls which were free of the disease. They were individually matched to the cases by age ( \pm 5 years). Ratio of cases and controls was 1:2. A specifically designed questionnaire was used to collect information on possible risk factors of type 2 diabetes. Smoking was assessed according to: smoking habits, duration of smoking, number of cigarettes smoked per day, pack years, smoking cessation. Anthropometrical measurements were made according to the guidelines of WHO. A conditional logistic regression was used to calculate odds ratio (OR) and corresponding $95 \%$ confidence interval $(\mathrm{Cl})$ for diabetes mellitus in relation to exposures of interest. Variables (such as family history on diabetes, body mass index (BMI), waist circumference (WC), education, plasma triglycerides (TG), morning exercise, eating speed, occupational and marital statuses) were retained in models as confounders when their inclusion changed the value of the OR by more than $5 \%$ in any exposure category. All the calculations were performed with the standard STATA 7 software program.

\section{Results}

The cases had higher BMI and significantly lower education level, compared to the controls (Table 1). After adjustment for possible confounders increased risk of type 2 diabetes was determined for those, whose smoked 10 and more cigarettes per day $(\mathrm{OR}=2.83 ; 95 \% \mathrm{Cl} 1.04-7.71$ vs. non-smokers). It has also been defined an association between the disease and duration of smoking (OR=4.55; $95 \% \mathrm{Cl} 1.11-18.61$ for 40 years or more smokers vs. non-smokers) and those, whose smoking cessation is 19 years and less had higher risk for diabetes $(\mathrm{OR}=6.40 ; 95 \% \mathrm{Cl} 1.50-27.34$ vs. non-smokers) (Table 2$)$.

Table 1. Characteristics of cases and controls.

\begin{tabular}{|c|c|c|c|c|c|c|}
\hline \multirow{2}{*}{ Variable } & \multirow{2}{*}{ Category } & \multicolumn{2}{|c|}{ Cases } & \multicolumn{2}{|c|}{ Controls } & \multirow{2}{*}{ p value } \\
\cline { 3 - 6 } & & $\mathrm{n}$ & $\%$ & $\mathrm{n}$ & $\%$ & \\
\hline Age & $\leq 44$ & 77 & 4.17 & 14 & 4.17 & \\
(years) & $45-54$ & 16 & 9.52 & 32 & 9.52 & \\
& $55-64$ & 66 & 39.29 & 130 & 38.69 & \\
& $\geq 65$ & 79 & 47.02 & 160 & 47.62 & matched \\
\hline Education & $\leq 10$ & 90 & 53.57 & 120 & 35.71 & \\
(years of & $11-13$ & 46 & 27.38 & 142 & 42.26 & \\
education) & $\geq 14$ & 32 & 19.05 & 74 & 22.02 & $<0.0001$ \\
\hline Marital & Married & 84 & 50.00 & 177 & 52.68 & \\
status & Divorced/separated & 15 & 8.93 & 37 & 11.01 & \\
& Single & 9 & 5.36 & 24 & 7.14 & \\
& Widowed & 60 & 35.71 & 98 & 29.17 & NS \\
\hline \multirow{4}{*}{ BMI } & $\leq 24.9$ & 12 & 7.14 & 84 & 25.00 & \\
& $25-29.9$ & 40 & 23.81 & 116 & 34.52 & \\
& $\geq 30.0$ & 116 & 69.05 & 136 & 40.48 & $<0.0001$ \\
\hline Family history & No & 118 & 70.24 & 297 & 88.39 & \\
of diabetes & Yes & 50 & 29.76 & 39 & 11.61 & $<0.0001$ \\
\hline
\end{tabular}

NS - not significant.
Table 2. Odds ratios and $95 \% \mathrm{Cl}$ confidence intervals for type 2 diabetes mellitus in relation to smoking

\begin{tabular}{|c|c|c|c|c|c|c|c|}
\hline \multirow[t]{2}{*}{ Variable } & \multirow[t]{2}{*}{ Category } & \multicolumn{2}{|c|}{ Cases } & \multicolumn{2}{|c|}{ Controls } & \multirow{2}{*}{$\begin{array}{l}\mathrm{OR}^{\star} \\
(95 \%) \\
\mathrm{p} \text { for trend }\end{array}$} & \multirow{2}{*}{$\begin{array}{c}\mathrm{OR}^{\star \star} \\
(95 \% \mathrm{Cl}) \\
\mathrm{p} \text { for trend }\end{array}$} \\
\hline & & $\mathrm{N}$ & $\%$ & $\mathrm{~N}$ & $\%$ & & \\
\hline \multirow[t]{3}{*}{ Smoking } & \multirow{3}{*}{ 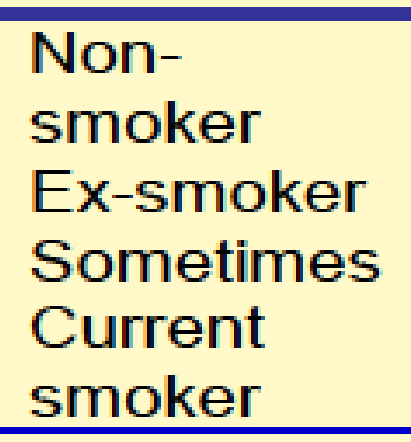 } & 137 & 81.55 & 300 & 89.29 & 1.00 & 1.00 \\
\hline & & $\begin{array}{c}16 \\
4\end{array}$ & $\begin{array}{l}9.52 \\
2.38\end{array}$ & $\begin{array}{c}18 \\
6\end{array}$ & $\begin{array}{l}5.36 \\
1.78\end{array}$ & $\begin{array}{l}1.68(0.79-3.60) \\
1.50(0.34-6.64)\end{array}$ & $\begin{array}{l}1.41(0.84-4.33) \\
1.14(0.23-5.59)\end{array}$ \\
\hline & & 11 & 6.55 & 12 & 3.57 & $\begin{array}{c}1.80(0.62-5.22) \\
p=0.281\end{array}$ & $\begin{array}{c}1.63(0.54-4.94) \\
p=0.384\end{array}$ \\
\hline \multirow{4}{*}{$\begin{array}{l}\text { Duration of } \\
\text { smoking } \\
\text { (years) }\end{array}$} & $\begin{array}{l}\text { Non- } \\
\text { smoker }\end{array}$ & 137 & 81.55 & 300 & 89.29 & 1.00 & 1.00 \\
\hline & $\leq 19$ & 7 & 4.16 & 17 & 5.06 & $0.71(0.26-1.94)$ & $0.92(0.32-2.60)$ \\
\hline & 20-39 & 11 & 6.55 & 15 & 4.46 & $\begin{array}{l}1.72(0.6 \\
0\end{array}$ & $\begin{array}{l}1.63(0.5 \\
1.55\end{array}$ \\
\hline & $\geq 40$ & 13 & 7.74 & 4 & 1.19 & $\begin{array}{c}5.70(1.50-2.1169 \\
p=0.011\end{array}$ & $\begin{array}{c}4.55(1.11-18.61) \\
p=0.035\end{array}$ \\
\hline \multirow{3}{*}{$\begin{array}{l}\text { Number } \\
\text { cigarettes } \\
\text { smoked per } \\
\text { day }\end{array}$} & $\begin{array}{l}\text { Non- } \\
\text { smoker }\end{array}$ & 137 & 81.55 & 300 & 89.29 & 1.00 & 1.00 \\
\hline & $\leq 9$ & 13 & 7.74 & 25 & 7.44 & $1.94(0.5)$ & $1.25(0.5$ \\
\hline & $\geq 10$ & 18 & 10.71 & 11 & 3.27 & $\begin{array}{c}2.93(1.1 \\
p=0 .\end{array}$ & $\begin{array}{c}2.83(1.0 \\
p=0 .\end{array}$ \\
\hline \multirow[t]{3}{*}{ Pack-years } & \multirow{3}{*}{$\begin{array}{l}\text { Non- } \\
\text { smoker } \\
\leq 9 \\
\geq 10\end{array}$} & 137 & 81.55 & 300 & 89.29 & 1.00 & 1.00 \\
\hline & & 18 & 10.71 & 31 & 9.22 & $1.19(0.60-2.40)$ & $1.38(0.66-2.88)$ \\
\hline & & 13 & 7.74 & 5 & 1.49 & $\begin{array}{r}4.83(1.4 \\
p=0\end{array}$ & $\begin{array}{c}3.29(0.87-12.42) \\
p=0.078\end{array}$ \\
\hline \multirow{3}{*}{$\begin{array}{l}\text { Smoking } \\
\text { cessation } \\
\text { (years) }\end{array}$} & \multirow{2}{*}{$\begin{array}{l}\text { Non- } \\
\text { smoker } \\
\leq 19\end{array}$} & 137 & 81.55 & 300 & 94.34 & & \\
\hline & & 10 & 6.54 & 4 & 1.26 & $6.60(1.67-26.05)$ & $6.40(1.50-27.34)$ \\
\hline & $\geq 20$ & 6 & 3.92 & 4.40 & 11.96 & $\begin{array}{c}0.94(0.32-2.77) \\
p=0.007\end{array}$ & $\begin{array}{c}1.18(0.39-3.59) \\
p=0.012\end{array}$ \\
\hline
\end{tabular}

$\mathrm{OR}^{*}$ adjusted for family history on diabetes and $\mathrm{BMI} ; \mathrm{OR}^{\star *}$ family history on diabetes, $\mathrm{BMI}$, waist circumference, education, plasma TG, morning exercise, eating speed, occupational and marital statuses.

\section{Conclusions}

Our data support a possible relationship between smoking and the increased risk of type 2 diabetes in women.

References:

1. van Dieren S, Beulens JW, van der Schouw YT et al. The global burden of diabetes and its complications: an emerging pandemic. European Journal of Cardiovascular Prevention \& Rehabilitation. 2010; 17: S3-S8.

2. Cornelis MC, Hu FB. Gene-environment interactions in the development of type 2 diabetes: recent progress and continuing challenges. Annual Review of Nutrition. 2012; 32: 245-259.

3. Ford SK, Shilliday BB. Smoking and diabetes: helping patients quit. Clin Diabetes. 2006; 24:133-137.

5.WHO. Available at: http://www.who.int/features/factfiles/tobacco_epidemic/tobacco_epidemic_facts/en/ Accessed January $28,2015$. 